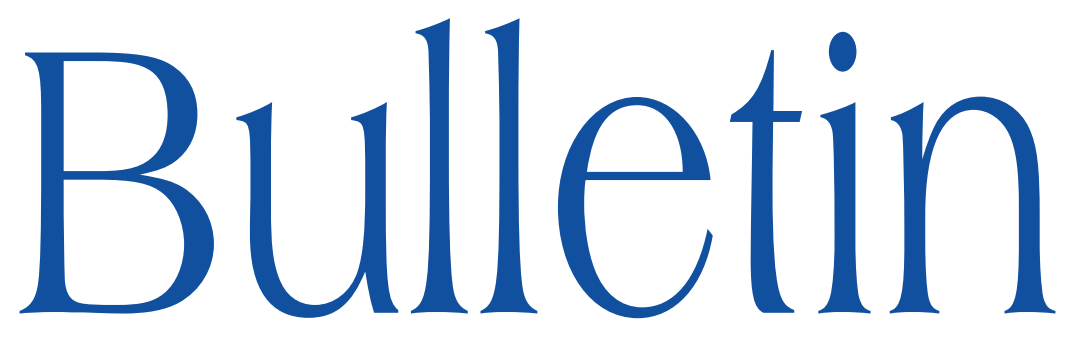

de la SOCIÉTÉ MATHÉMATIQUE DE FRANCE

\title{
CONSTANTES DE SOBOLEV DES ARBRES
}

\author{
Marc Bourdon
}

\section{Tome 135} Fascicule 1

2007 


\title{
CONSTANTES DE SOBOLEV DES ARBRES
}

\author{
PAR MARC BOURDON
}

RÉsumé. - Étant donnés $p \in[1,+\infty$ [ et un arbre $T$ dont chaque sommet est de valence au moins 3, on étudie la constante de Sobolev d'exposant $p$ de $T$, c'est-à-dire la plus petite constante $\sigma_{p}$ telle que pour tout $u \in \ell_{p}\left(T^{0}\right)$ on ait $\|u\|_{p}^{p} \leq \sigma_{p}\|\mathrm{~d} u\|_{p}^{p}$. Notre motivation vient de la recherche de graphes finis avec des petites constantes de Poincaré d'exposant $p$, en vue d'obtenir des exemples de groupes qui ont la propriété de point fixe sur les espaces $L^{p}$.

Abstract (Sobolev constants for trees). - For $p \in[1,+\infty[$ and for any tree $T$ of valency at least 3 , we study the Sobolev constant of exponent $p$ of $T$, that is the smallest constant $\sigma_{p}$ such that for every $u \in \ell_{p}(T)$, one has $\|u\|_{p}^{p} \leq \sigma_{p}\|\mathrm{~d} u\|_{p}^{p}$. Our motivation comes from the search of finite graphs with small Poincare constants of exponent $p$, in order to construct examples of groups which admit the fixed point property on $L^{p}$-spaces.

\section{Introduction}

Soit $G$ un graphe fini ; notons $G^{0}$ l'ensemble de ses sommets et $G^{1}$ l'ensemble de ses arêtes non orientées. Pour $p \in] 1,+\infty[$ on s'intéresse à la plus petite

Texte reçu le 23 janvier 2006, révisé le 6 avril 2007

MARc Bourdon, Laboratoire Paul Painlevé, UMR CNRS 8524, UFR de Mathématiques Pures et Appliquées, Bât. M2, Université des Sciences et Technologies de Lille, 59665 Villeneuve d'Ascq Cedex (France) - E-mail : Marc.Bourdon@math.univ-lille1.fr Classification mathématique par sujets (2000). - 58E35, 31C45.

Mots clefs. - Constantes de Sobolev, constantes de Poincaré, arbres, graphes. 
constante $\pi_{p}$ telle que pour toute fonction réelle $u$ de $G^{0}$ on ait

$$
\sum_{x \in G^{0}}\left|u(x)-b_{u}\right|^{p} \leq \pi_{p} \sum_{a \in G^{1}}|\mathrm{~d} u(a)|^{p},
$$

où $b_{u}$ est le « $p$-barycentre » des $u(x),\left(x \in G^{0}\right)$, c'est-à-dire l'unique $b \in \mathbb{R}$ qui minimise la fonction

$$
t \longmapsto \sum_{x \in G^{0}}|u(x)-t|^{p},
$$

et où $\mathrm{d} u$ désigne la différentielle de $u$ : pour toute arête $a$ d'extrémités $a_{+}$et $a_{-}$ dans $G^{0}$,

$$
|\mathrm{d} u(a)|=\left|u\left(a_{+}\right)-u\left(a_{-}\right)\right| .
$$

La constante $\pi_{p}$ s'appelle la constante de Poincaré d'exposant $p$ du graphe $G$. Son inverse $\pi_{p}^{-1}$ est égale à la plus petite «valeur propre » non nulle du $p$ Laplacien de $G$ (voir 1.2), c'est-à-dire de l'opérateur défini sur les fonctions $u$ de $G^{0}$ par

$$
\forall x \in G_{0}, \quad\left(\Delta_{p} u\right)(x)=\sum_{y \sim x}|u(x)-u(y)|^{p-2}(u(x)-u(y)) .
$$

Pour $p=2$, l'étude de $\pi_{p}$ est bien développée (voir [8] et les références). Par contre, lorsque $p$ est grand, même pour les graphes les plus simples, il est difficile d'estimer $\pi_{p}$ et le sujet semble très ouvert.

On étudie dans cette note le problème analogue pour les arbres. Soit $T$ un arbre dont chaque sommet est de valence au moins égale à 3 . Pour $p \in[1,+\infty[$ soit $\sigma_{p}$ la plus petite constante telle que pour toute fonction réelle $u$ de $T^{0}$ $p$-sommable, on ait

$$
\sum_{x \in T^{0}}|u(x)|^{p} \leq \sigma_{p} \sum_{a \in T^{1}}|\mathrm{~d} u(a)|^{p} .
$$

Elle s'appelle la constante de Sobolev d'exposant $p$ de $T$. On démontre :

\section{THÉORÈME 1.1}

a) Si T est homogène de valence $k$, alors

$$
\sigma_{p}=\left[(k-1)^{\frac{1}{p}}-1\right]^{-p} .
$$

b) Si la valence de chaque sommet de $T$ appartient $\grave{a}[3, k]$, alors

$$
\sigma_{p} \geq\left[(k-1)^{\frac{1}{p}}-1\right]^{-p} .
$$

c) Si la valence de chaque sommet de $T$ est supérieure ou égale à $k \geq 3$, alors

$$
\sigma_{p} \leq\left[(k-1)^{\frac{1}{p}}-1\right]^{-p} .
$$

Pour $p=2$ ces relations sont classiques (voir [8]), dans le cas général elles ne semblent pas apparaître dans la littérature.

TOME $135-2007-\mathrm{N}^{\mathrm{O}} 1$ 
Remarques et motivations a) La constante de Poincaré joue un rôle important dans la mise en œuvre par M. Gromov de la méthode de Garland pour obtenir des théorèmes de points fixes (voir [5, 4]). En particulier, il découle de [5] le résultat suivant, qui généralise à $p \neq 2$ un théorème d'A. Zuk [10] et de Ballmann-Swiatkowski [2] (voir aussi [9, 7, 1] pour des résultats voisins) :

Soit $X$ un 2-complexe simplicial connexe, de valence constante égale à $k \geq 3$ (i.e. toute arête de $X$ est commune à $k$ triangles de $X$ ) et soit $\Gamma$ un groupe agissant sur $X$ de manière simpliciale, libre, et cocompacte. On suppose que deux triangles distincts quelconques de $X / \Gamma$ ont au plus un sommet ou une arête en commun. Soit $\mu$ une mesure borélienne sur $\mathbb{R}$ et soit $p \in] 1,+\infty[$. Alors si les constantes de Poincaré d'exposant $p$ des links des sommets de $X$ sont toutes strictement inférieures à $2 / k$, toute action isométrique de $\Gamma$ sur $L^{p}(\mathbb{R}, \mu)$ possède un point fixe global.

Ce résultat implique en particulier l'annulation du premier groupe de cohomologie $\ell_{p}$ de $\Gamma$, c'est à dire le $H^{1}\left(\Gamma, \ell_{p}(\Gamma)\right)$. En effet il suffit de prendre pour $\mu$ la mesure de comptage d'un sous-ensemble dénombrable de $\mathbb{R}$.

b) Le théorème 1.1 montre que pour $p$ fixé, la constante de Sobolev $\sigma_{p}$ de l'arbre régulier de valence $k$ est équivalente à $1 / k$ lorsque $k$ tend vers l'infini. On peut donc espérer que pour $p>1$ et $c>1$ fixées, il existe de nombreux graphes finis de valence $k$ qui satisfont $\pi_{p} \leq c / k$. J'ignore toutefois comment construire de tels graphes (voir [8] pour $p=2$ ).

c) Décrivons les cas limites $p=1$ et $=\infty$ de l'inégalité $\left(I_{p}\right)$. La constante $\pi_{1}$ est également (par des arguments standards, voir [3]), la plus petite constante telle que pour tout $A \subset G^{0}$ avec $|A| \leq \frac{1}{2}\left|G^{0}\right|$, on ait

$$
|A| \leq \pi_{1}|\partial A|
$$

où $\partial A$ désigne l'ensemble des $a \in G^{1}$ qui ont une extrémité dans $A$ et l'autre dans $A^{c}$.

Posons

$$
\pi_{\infty}=\lim _{p \rightarrow+\infty} \pi_{p}^{1 / p}
$$

Alors $\pi_{\infty}$ est la plus petite constante telle que pour toute fonction $u$ de $G^{0}$, on ait

$$
\frac{1}{2}(\sup u-\inf u) \leq \pi_{\infty} \sup |\mathrm{d} u| .
$$

Il est tentant de croire que la fonction $\theta \mapsto \theta \log \pi_{1 / \theta}$ est convexe sur $[0,1]$, comme dans le théorème d'interpolation de Riesz. 
Remerciements. - Je remercie Yann Ollivier dont les questions sont à l'origine de cette note. Merci également à Frédéric Haglund, Hiroyasu Izeki, Pierre Pansu, et Shin Nayatani pour leurs intérêt et suggestions. Ce travail a été effectué lors d'un séjour aux instituts de mathématiques de Chennai et d'Allahabad, je remercie ces institutions pour leur hospitalité. Enfin je remercie le rapporteur qui m'a suggéré d'énoncer et de démontrer les inégalités b) et c) du théorème 1.1 .

\section{Quelques lemmes}

Pour un ensemble $E$ et $p \in] 1,+\infty\left[\right.$, on note $\ell_{p}(E)$ l'ensemble des fonctions réelles de $E$ qui sont $p$-sommables et $\|\cdot\|_{p}$ leur $p$-norme. Soit $G$ un graphe connexe de valence uniformément bornée. On suppose que les extrémités de chaque arête sont distinctes et que deux arêtes qui ont mêmes extrémités sont confondues. Pour $u \in \ell_{p}\left(G^{0}\right)$, sa $p$-énergie est le nombre $\|\mathrm{d} u\|_{p}^{p}$. Supposons $G$ infini, et considérons l'inverse de la constante de Sobolev de $G$

$$
\lambda:=\inf \left\{\frac{\|\mathrm{d} u\|_{p}^{p}}{\|u\|_{p}^{p}} ; u \in \ell_{p}\left(G^{0}\right) \backslash\{0\}\right\} .
$$

En général, l'infimum n'est pas atteint, les lemmes qui suivent permettent de se ramener à la dimension finie. Pour un sous-espace $\mathcal{E}$ de $\ell_{p}\left(G^{0}\right)$, on note

$$
\lambda_{\mathcal{E}}=\inf \left\{\frac{\|\mathrm{d} u\|_{p}^{p}}{\|u\|_{p}^{p}} ; u \in \mathcal{E} \backslash\{0\}\right\} .
$$

Soit $H$ un sous-graphe fermé de $G$, on dénote par $\mathcal{E}_{H}$ le sous-espace de $\ell_{p}\left(G^{0}\right)$ constitué des fonctions nulles en dehors de $H^{0}$, et par $\mathcal{P}_{H}$ la projection de $\ell_{p}\left(G^{0}\right)$ sur $\mathcal{E}_{H}$. Le lemme suivant est immédiat.

Lemme 2.1. - Soit $\left\{G_{n}\right\}$ une suite de sous-graphes fermés de $G$, croissante, et telle que l'union des $G_{n}$ soit égale à $G$. Soit $\mathcal{E}$ un sous-espace de $\ell_{p}\left(G^{0}\right)$ et soit $\mathcal{E}_{n}$ son image par $\mathcal{P}_{G_{n}}$. Alors

$$
\lim _{n \rightarrow+\infty} \lambda_{\mathcal{E}_{n}}=\lambda_{\mathcal{E}}
$$

Rappelons que le $p$-Laplacien de $G$ est l'application de $\left\{u: G^{0} \rightarrow \mathbb{R}\right\}$ dans lui-même définie par

$$
\forall x \in G_{0}, \quad\left(\Delta_{p} u\right)(x)=\sum_{y \sim x}\{u(x)-u(y)\}^{p-1},
$$

où $y \sim x$ signifie que $x$ et $y$ sont les extrémités d'une arête de $G$, et où $\{t\}^{p-1}$ désigne $t^{p-1}$ si $t \geq 0$ et $-|t|^{p-1}$ sinon. On renvoie à [6] et à ses références pour l'analyse $p$-harmonique.

Désignons par $\langle h, k\rangle$ le produit scalaire $\sum_{x \in G^{0}} h(x) k(x)$.

TOME $135-2007-\mathrm{N}^{\circ} 1$ 
LEMME 2.2. - Soit $\mathcal{F}$ un sous-espace de dimension finie de $\ell_{p}\left(G^{0}\right)$. Alors $\lambda_{\mathcal{F}}$ est égale au minimum des $\alpha \in \mathbb{R}$ pour lesquels il existe $u \in \mathcal{F}, u \neq 0$, avec

$$
\forall v \in \mathcal{F}, \quad\left\langle v, \Delta_{p} u-\alpha\{u\}^{p-1}\right\rangle=0 .
$$

Démonstration. - Puisque $\mathcal{F}$ est de dimension finie, $\lambda_{\mathcal{F}}$ est la plus petite valeur critique de la fonction de $\mathcal{F}$,

$$
\Psi(u)=\frac{\|\mathrm{d} u\|_{p}^{p}}{\|u\|_{p}^{p}}
$$

Posons $\varphi(u)=\|\mathrm{d} u\|_{p}^{p}$. On a pour $h \in \ell_{p}\left(G^{0}\right)$

$$
D_{u} \Psi(h)=\frac{1}{\|u\|_{p}^{p}} D_{u} \varphi(h)-\frac{p}{\|u\|_{p}^{2 p}}\left\langle h,\{u\}^{p-1}\right\rangle\|\mathrm{d} u\|_{p}^{p} .
$$

De plus, en notant $\vec{G}^{1}$ l'ensemble des arêtes orientées de $G$, on a :

$$
\begin{aligned}
D_{u} \varphi(h) & =p \sum_{[x y] \in G^{1}}\{u(x)-u(y)\}^{p-1}(h(x)-h(y)) \\
& =\frac{1}{2} p \sum_{[\overrightarrow{x y}] \in \vec{G}^{1}}\{u(x)-u(y)\}^{p-1}(h(x)-h(y)) \\
& =p \sum_{[\vec{x}] \in \vec{G}^{1}} h(x)\{u(x)-u(y)\}^{p-1} \\
& =p \sum_{x \in G^{0}} h(x) \sum_{y \sim x}\{u(x)-u(y)\}^{p-1}=p\left\langle h, \Delta_{p} u\right\rangle .
\end{aligned}
$$

Finalement, on obtient

$$
D_{u} \Psi(h)=\frac{p}{\|u\|_{p}^{p}}\left\langle h, \Delta_{p} u-\Psi(u)\{u\}^{p-1}\right\rangle .
$$

Le lemme qui suit permet de réduire encore le domaine d'étude.

LEMme 2.3. - Soit $\pi: G \rightarrow \bar{G}$ une application simpliciale du graphe $G$ sur un graphe $\bar{G}$. Supposons qu'elle ait la propriété suivante : pour toute arête $\bar{a}=[\bar{x} \bar{y}]$ de $\bar{G}$ et toute préimage $x$ de $\bar{x}$, le nombre de voisins de $x$ dans $\pi^{-1}(\bar{y})$ ne dépend pas de $x$ mais seulement de $\bar{a}$ et de $\bar{x}$. Supposons aussi que toutes les fibres de $\pi$ soient des ensembles finis. Soit $\mathcal{E}$ le sous-espace de $\ell_{p}\left(G^{0}\right)$ constitué des fonctions de $G^{0}$ constantes sur chaque fibre de $\pi$. Alors $\lambda=\lambda_{\mathcal{E}}$.

Démonstration. - Montrons que $\lambda_{\mathcal{E}} \leq \lambda$, l'autre inégalité étant triviale. Étant donnée $u \in \ell_{p}\left(G^{0}\right)$, on définit $v \in \mathcal{E}$ comme suit : pour $x \in G^{0}$, notons $F_{x}$ la fibre de $\pi$ qui contient $x$, et posons

$$
v(x)=\left(\frac{1}{\left|F_{x}\right|} \sum_{z \in F_{x}}|u(z)|^{p}\right)^{1 / p} .
$$


La $p$-norme de $v$ est égale à celle de $u$. Montrons que la $p$-énergie de $v$ est inférieure à celle de $u$, le lemme en découlera. Soit $[\bar{x} \bar{y}]$ une arête de $\bar{G}$ et soit respectivement $F, F_{-}$et $F_{+}$l'ensemble des préimages de $[\bar{x} \bar{y}], \bar{x}$ et $\bar{y}$. On a :

$$
\begin{gathered}
\left(\frac{1}{|F|} \sum_{[x y] \in F}|v(x)-v(y)|^{p}\right)^{1 / p}=|v(x)-v(y)| \\
=\left|\left(\frac{1}{\left|F_{-}\right|} \sum_{x \in F_{-}}|u(x)|^{p}\right)^{1 / p}-\left(\frac{1}{\left|F_{+}\right|} \sum_{y \in F_{+}}|u(y)|^{p}\right)^{1 / p}\right| \\
=\left|\left(\frac{1}{|F|} \sum_{[x y] \in F}|u(x)|^{p}\right)^{1 / p}-\left(\frac{1}{|F|} \sum_{[x y] \in F}|u(y)|^{p}\right)^{1 / p}\right| \\
\leq\left(\frac{1}{|F|} \sum_{[x y] \in F}|u(x)-u(y)|^{p}\right)^{1 / p} .
\end{gathered}
$$

En effet, la troisième égalité provient de l'hypothèse sur le nombre de voisins des éléments de $F_{-}$dans $F_{+}$, et la dernière inégalité est l'inégalité de Minkowski. Ainsi, on obtient :

$$
\sum_{[x y] \in F}|v(x)-v(y)|^{p} \leq \sum_{[x y] \in F}|u(x)-u(y)|^{p},
$$

puis $\|\mathrm{d} v\|_{p}^{p} \leq\|\mathrm{d} u\|_{p}^{p}$ en sommant l'inégalité sur toutes les arêtes de $\bar{G}$.

Comme exemples d'applications $\pi$ qui satisfont les conditions du lemme 2.3, on trouve les revêtements finis et les quotients de $G$ par un groupe compact d'isométries.

\section{Cas des arbres homogènes}

On démontre dans ce chapitre l'égalité a) du théorème 1.1 de l'introduction.

Soit $I$ le graphe porté par $[0,+\infty]$ dont les sommets sont les nombres entiers. Soit $T$ l'arbre homogène de valence $k \geq 3$ et $O$ un de ses sommets. Le quotient de $T$ par le groupe des isométries de $T$ qui fixe $O$ est isomorphe au graphe $I$. L'application quotient $\pi: T \rightarrow I$ satisfait aux hypothèses du lemme 2.3. Notons $\mathcal{E}$ le sous-espace de $\ell_{p}\left(T^{0}\right)$ constitué des fonctions constantes sur chaque fibre de $\pi$. Soit aussi $T_{n}=\pi^{-1}([0, n])$ pour $n \in \mathbb{N}$, et soit $\mathcal{E}_{n}=\mathcal{P}_{T_{n}}(\mathcal{E})$. Notons $\lambda_{n}$ la constante $\lambda_{\mathcal{E}_{n}}$. D'après les lemmes 2.1 et 2.3 , on a

$$
\lim _{n \rightarrow+\infty} \lambda_{n}=\lambda_{\mathcal{E}}=\lambda \text {. }
$$

D'après le lemme 2.2, la constante $\lambda_{n}$ est la plus petite des constantes $\alpha$ pour lesquelles il existe $u \in \mathcal{E} \backslash\{0\}$ qui satisfait

$$
\forall x \in T_{n}^{0}, \quad\left(\Delta_{p} u\right)(x)=\alpha\{u\}^{p-1} \text { et } \forall x \in T^{0} \backslash T_{n}^{0}, u(x)=0 .
$$


Observons que les fonctions $u$ et $|u|$ ont même $p$-norme et que la $p$-énergie de $|u|$ est inférieure ou égale à celle de $u$. Donc, en plus des conditions précédentes, on peut imposer $u \geq 0$. En calculant le $p$-laplacien sur les éléments de $\mathcal{E}$ on se ramène à chercher le plus petit $\alpha \in \mathbb{R}$ pour lequel il existe $v: \mathbb{N} \rightarrow \mathbb{R}^{+}$ non identiquement nulle avec

(i) $k\{v(0)-v(1)\}^{p-1}=\alpha\{v(0)\}^{p-1}$

(ii) pour tout $\ell \in\{1,2, \ldots, n\}$,

$$
(k-1)\{v(\ell)-v(\ell+1)\}^{p-1}+\{v(\ell)-v(\ell-1)\}^{p-1}=\alpha\{v(\ell)\}^{p-1},
$$

(iii) pour tout $\ell \geq n+1, v(\ell)=0$.

Puisque $v$ et $\alpha$ sont positives, on voit par récurrence que $v$ est décroissante. Pour $\ell \in\{1, \ldots, n\}$, l'équation (ii) s'écrit encore

$$
(k-1)\left(1-\frac{v(\ell+1)}{v(\ell)}\right)^{p-1}-\left(\frac{v(\ell-1)}{v(\ell)}\right)^{p-1}\left(1-\frac{v(\ell)}{v(\ell-1)}\right)^{p-1}=\alpha .
$$

En posant

$$
w(\ell)=\left(1-\frac{v(\ell)}{v(\ell-1)}\right)^{p-1}
$$

elle devient

$$
w(\ell+1)=\frac{1}{k-1} \frac{w(\ell)}{\left(1-w(\ell)^{1 /(p-1)}\right)^{p-1}}+\frac{\alpha}{k-1} .
$$

Posons

$$
f_{\alpha}(t)=\frac{1}{k-1} \frac{t}{\left(1-t^{1 /(p-1)}\right)^{p-1}}+\frac{\alpha}{k-1} .
$$

La détermination de $\lambda_{n}$ se réduit à trouver le plus petit $\alpha>0$ pour lequel il existe $w$ défini de $\{1, \ldots, n+1\}$ dans $[0,1]$, avec

(i) $w(1)=\alpha / k$,

(ii) ${ }^{\prime}$ pour $\ell \in\{1, \ldots, n\}, w(\ell+1)=f_{\alpha}(w(\ell))$,

(iii) $)^{\prime} w(n+1)=1$.

Ces conditions sont équivalentes à

(iv) pour tout $\ell \in\{1, \ldots, n-1\}, f_{\alpha}^{\ell}(\alpha / k) \in[0,1]$ et $f_{\alpha}^{n}(\alpha / k)=1$.

Étudions $f_{\alpha}$ entre 0 et 1 . Elle s'écrit $f_{0}+\alpha /(k-1)$ : donc son graphe se déduit de celui de $f_{0}$ par une translation verticale. Un calcul élémentaire donne :

$$
f_{\alpha}^{\prime}(t)=\frac{1}{k-1} \frac{1}{\left(1-t^{1 /(p-1)}\right)^{p}} .
$$

Donc $f_{\alpha}$ est croissante et convexe sur $[0,1]$. Par un calcul l'équation $f_{\alpha}^{\prime}(t)=1$ possède dans $[0,1]$ l'unique solution $t_{0}=\left(1-\frac{1}{(k-1)^{1 / p}}\right)^{p-1}$. De plus $f_{\alpha}\left(t_{0}\right)=t_{0}$ si et seulement si $\alpha=\left((k-1)^{1 / p}-1\right)^{p}$; soit $\alpha_{0}$ ce nombre. Considérons les cas $\left.\alpha \in] 0, \alpha_{0}\right]$ et $\alpha>\alpha_{0}$. 
Lorsque $\left.\alpha \in] 0, \alpha_{0}\right]$, le graphe de $f_{\alpha}$ coupe la diagonale en deux points $t_{1}, t_{2}$ (confondus si $\alpha=\alpha_{0}$ ) avec $0<t_{1} \leq t_{0}<t_{2}<1$. Puisque

$$
\frac{\alpha}{k} \leq \frac{\alpha}{k-1}=f_{\alpha}(0) \leq f_{\alpha}\left(t_{1}\right)=t_{1},
$$

on a $f_{\alpha}^{\ell}(\alpha / k) \leq t_{1}$ pour tout $\ell \in \mathbb{N}^{*}$. Donc les conditions (iv) ne sont pas satisfaites et donc $\lambda_{n} \geq \alpha_{0}$. Par passage à la limite, il vient $\lambda \geq \alpha_{0}$.

Quand $\alpha>\alpha_{0}$, le graphe de $f_{\alpha}$ se situe au dessus de la diagonale. Donc pour $\ell$ assez grand on a $f_{\alpha}^{\ell}(\alpha / k) \geq 1$. Par ailleurs, on a

$$
\frac{\alpha_{0}}{k}<\frac{\alpha_{0}}{k-1}=f_{\alpha_{0}}(0) \leq f_{\alpha_{0}}\left(t_{0}\right)=t_{0} \text {. }
$$

Lorsque $\alpha$ proche de $\alpha_{0}$ l'inégalité $\alpha_{0} / k<t_{0}$ subsiste. Dès lors pour $\alpha$ proche de $\alpha_{0}$, les puissances $f_{\alpha}^{\ell}(\alpha / k)$ passent un long moment proches de $t_{0}$ avant d'être supérieures à 1. Par un argument de valeurs intermédiaires, les conditions (iv) sont satisfaites avec $\alpha$ arbitrairement proche de $\alpha_{0}$ en choisissant $n$ assez grand. D'où $\lambda \leq \alpha_{0}$, puis l'égalité.

\section{4. Élagage}

On montre dans ce chapitre les inégalités b) et c) du théorème 1.1 de l'introduction. Leur preuve utilisera les deux lemmes ci-dessous.

Soit $G$ un graphe quelconque comme au début du chapitre 2 , soit $x_{0}$ un sommet de $G$ et $n$ un entier, on note $\mathcal{E}_{G}\left(x_{0}, n\right)$ le sous-espace de $\ell_{p}\left(G^{0}\right)$ constitué des fonctions nulles en dehors de la boule fermée de centre $x_{0}$ et de rayon $n$. Soit aussi $\lambda_{G}\left(x_{0}, n\right)$ la constante associée définie au début du chapitre 2 . D'après le lemme 2.1, l'inverse de la constante de Sobolev de $G$, notée $\lambda_{G}$, satisfait

$$
\lambda_{G}=\lim _{n \rightarrow+\infty} \lambda_{G}\left(x_{0}, n\right) .
$$

LEMME 4.1. - Soit $u$ une fonction positive ou nulle appartenant à $\ell_{p}\left(G^{0}\right)$, qui satisfait pour tout $x \in G^{0}$ avec $u(x) \neq 0$,

$$
\Delta_{p} u(x) \leq \alpha\{u(x)\}^{p-1},
$$

alors $\|\mathrm{d} u\|_{p}^{p} \leq \alpha\|u\|_{p}^{p}$.

Démonstration. - Par un calcul déjà effectué dans la preuve du lemme 2.2, et puisque $u$ est positive ou nulle, on a

$$
\|\mathrm{d} u\|_{p}^{p}=\left\langle u, \Delta_{p} u\right\rangle \leq \alpha\left\langle u,\{u\}^{p-1}\right\rangle=\alpha\|u\|_{p}^{p} .
$$

DÉfinition 4.2. - Soit $A$ un arbre et $a=\left[a^{-} a^{+}\right]$une arête orientée de $A$. On appelle branche de $A$ issue de a la composante connexe de $A \backslash a^{-}$qui contient l'arête $a$. 
On décrit à présent un procédé d'élagage d'un arbre qui décroit l'inverse des constantes de Sobolev.

LEMme 4.3. - Soit $A$ un arbre de valence uniformément bornée, et soit u une fonction positive ou nulle dans $\mathcal{E}_{A}\left(x_{0}, n\right)$ qui réalise l'infimum $\lambda_{A}\left(x_{0}, n\right)$. Considérons une collection $\left\{a_{1}, \ldots, a_{k}\right\}$ d'arêtes orientées de A telle que :

(i) pour tout $i \in\{1, \ldots, k\}, u\left(a_{i}^{-}\right) \geq u\left(a_{i}^{+}\right)$,

(ii) les branches issues des $a_{i}$ sont deux à deux disjointes et ne contiennent pas $x_{0}$,

alors en notant $A^{\prime}$ l'arbre $A$ privé de l'union des branches issues des $a_{i}$, on a

$$
\lambda_{A^{\prime}}\left(x_{0}, n\right) \leq \lambda_{A}\left(x_{0}, n\right)
$$

Démonstration. - D'après le lemme 2.2, on a

$$
\Delta_{p} u(x)=\lambda_{A}\left(x_{0}, n\right)\{u(x)\}^{p-1}
$$

pour tout $x \in A^{0}$ avec $u(x) \neq 0$. Soit $u^{\prime}$ la restriction de $u$ à l'ensemble des sommets de $A^{\prime}$. Compte tenu de l'hypothèse (i), pour tout $x \in A^{\prime 0}$ avec $u^{\prime}(x) \neq 0$, on a

$$
\Delta_{p} u^{\prime}(x) \leq \Delta_{p} u(x)=\lambda_{A}\left(x_{0}, n\right)\left\{u^{\prime}(x)\right\}^{p-1} .
$$

Donc le lemme 4.1 donne $\left\|\mathrm{d} u^{\prime}\right\|_{p}^{p} \leq \lambda_{A}\left(x_{0}, n\right)\left\|u^{\prime}\right\|_{p}^{p}$. Puisque, d'après l'hypothèse (ii), $u^{\prime}$ appartient à $\mathcal{E}_{A^{\prime}}\left(x_{0}, n\right)$, on obtient l'inégalité cherchée.

Passons à la

Preuve des inégalités b) et c) du théorème 1.1. - Prouvons d'abord l'inégalité $\mathrm{b})$. Soit $A$ un arbre dont les sommets sont de valences comprises entre 3 et $k$, avec $k \geq 3$, et soit $x_{0}$ un sommet de $A$. Notons $T$ l'arbre homogène de valence $k$. On construit l'espace pointé $\left(A, x_{0}\right)$ à partir de $T$ en choisissant une origine encore notée $x_{0}$ de $T$, puis en privant $T$ d'une collection de branches disjointes qui sont de plus en plus éloignées de $x_{0}$. D'après le chapitre 3 , la fonction positive ou nulle $u$ de $\mathcal{E}_{T}\left(x_{0}, n\right)$ qui réalise $\lambda_{T}\left(x_{0}, n\right)$, est décroissante le long des rayons géodésiques de $T$ issus de $x_{0}$. Ainsi les hypothèses du lemme 4.3 sont satisfaites, et donc $\lambda_{A}\left(x_{0}, n\right) \leq \lambda_{T}\left(x_{0}, n\right)$. En faisant tendre $n$ vers l'infini on obtient l'inégalité b).

Prouvons l'inégalité c). Soit $A$ un arbre dont les sommets sont de valences supérieures ou égales à $k$ avec $k \geq 3$. On va montrer par récurrence sur le cardinal des boules fermées $B\left(x_{0}, n\right)$ de $A^{0}$ que, pour tout arbre $A$ comme cidessus, pour tout entier $n$ et pour tout sommet $x_{0}$ de $A$, on a $\lambda_{T} \leq \lambda_{A}\left(x_{0}, n\right)$. En faisant tendre $n$ vers l'infini on en déduit l'inégalité c).

Tout d'abord si le cardinal de $B\left(x_{0}, n\right)$ est égal à $k+1$, alors $n=1$ et $B\left(x_{0}, n\right)$ est isométrique à la boule de rayon 1 de $T$. Dans ce cas l'inégalité 
cherchée est trivialement vraie (noter que $\lambda_{T}\left(x_{0}, n\right)$ est une suite décroissante de $n$ ).

Supposons donc l'inégalité vraie pour toutes les boules de cardinal inférieur strictement à $N$, et montrons qu'elle est vraie pour les boules de cardinal $N$. Soit $B=B\left(x_{0}, n\right)$ une boule de cardinal $N$ dans un arbre $A$ comme ci-dessus. Si tous les sommets intérieurs de $B$ sont de valence $k$, alors $B$ est isométrique à la boule de rayon $n$ de $T$, et comme dans le premier cas l'inégalité est trivialement vraie. Sinon il existe au moins un sommet $x_{1}$, intérieur à $B$, de valence supérieure ou égale à $k+1$. Soit $u$ une fonction dans $\mathcal{E}_{A}\left(x_{0}, n\right)$ qui réalise $\lambda_{A}\left(x_{0}, n\right)$. Quitte à considérer la fonction $|u|$, on peut supposer que $u$ est positive ou nulle. D'après le lemme 2.2, elle satisfait en tout sommet $x$ de $B$ l'égalité $\Delta_{p} u(x)=$ $\lambda_{A}\left(x_{0}, n\right)\{u(x)\}^{p-1}$. On en déduit que $x_{1}$ possède au moins un sommet voisin $y$ dans $B$ avec $u\left(x_{1}\right)-u(y) \geq 0$. Soit $a$ l'arête orientée $\left[x_{1} y\right]$.

Si elle ne pointe pas vers $x_{0}$, on applique le lemme $4.3:$ l'arbre $A^{\prime}$ obtenu en privant $A$ de la branche issue de $a$ satisfait $\lambda_{A^{\prime}}\left(x_{0}, n\right) \leq \lambda_{A}\left(x_{0}, n\right)$; de plus sa boule de centre $x_{0}$ et de rayon $n$ est de cardinal strictement inférieur à $N$. Avec l'hypothèse de récurrence on obtient l'inégalité cherchée.

Sinon, l'arête $a$ pointe vers $x_{0}$. On considère encore l'arbre $A^{\prime}$ obtenu en privant $A$ de la branche issue de $a$. La restriction $u^{\prime}$ de $u$ à $A^{\prime 0}$ appartient à $\mathcal{E}_{A^{\prime}}\left(x_{1}, n-\ell\right)$, où $\ell$ est distance dans $A$ entre $x_{0}$ et $x_{1}$. De plus $u^{\prime}$ satisfait les hypothèses du lemme 4.1 avec $\alpha=\lambda_{A}\left(x_{0}, n\right)$. Par suite le lemme 4.1 implique

$$
\lambda_{A^{\prime}}\left(x_{1}, n-\ell\right) \leq \lambda_{A}\left(x_{0}, n\right) .
$$

La boule de $A^{\prime}$ de centre $x_{1}$ et de rayon $n-\ell$ est de cardinal strictement inférieur à $N$, donc avec l'hypothèse de récurrence on obtient l'inégalité cherchée.

\section{BIBLIOGRAPHIE}

[1] U. Bader, A. Furman, T. Gelander \& N. Monod - «Property $(T)$ and rigidity for actions on Banach spaces », Acta Math. 198 (2007), p. 57105.

[2] W. Ballmann \& J. Swiatkowski - « On $L^{2}$-cohomology and property $(T)$ for automorphism groups of polyedral cell complexes », Geom. Funct. Anal. 7 (1997), p. 615-645.

[3] I. ChAVel - «Isoperimetric inequalities. Differential geometric and analytic perspectives », Cambridge Tracts in Math. 145 (2001).

[4] É. GHYs - «Groupes aléatoires (d'après Misha Gromov,...) », in Séminaire Bourbaki, vol. 2002/2003, Astérisque, vol. 294, Soc. Math. France, 2004, mars 2003, exp. no 916 .

TOME $135-2007-\mathrm{N}^{\circ} 1$ 
[5] M. Gromov - «Random walk in random groups », Geom. Funct. Anal. 13 (2003), p. 73-146.

[6] I. Holopainen \& P. Soardi - «p-harmonic functions on graphs and manifolds », Manuscripta Math. 94 (1997), p. 95-110.

[7] H. IZeKi \& S. NAYATANI - « Combinatorial harmonic maps and discretegroup actions on Hadamard spaces », Geom. Dedicata 114 (2005), p. 147188.

[8] A. Lubotzky - Discrete groups, expanding graphs and invariant measures, Progr. Math., vol. 125, 1994.

[9] P. PANSU - «Formules de Matsushima, de Garland et propriété $(T)$ pour des groupes agissant sur des espaces symétriques ou des immeubles », Bull. Soc. Math. France 126 (1998), p. 107-139.

[10] A. ŻuK - «La propriété $(T)$ de Kazhdan pour les groupes agissant sur les polyèdres », C. R. Acad. Sci. Paris, Sér. I 323 (1996), p. 453-458. 